\title{
La ciudad construida y filmada. Influencia de la Arquitectura Mo- derna en el imaginario distópico de Gattaca y Blade Runner 2049
}

\section{The Built and Filmed City. Influence of Modern Architecture in the dystopian imaginary of Gattaca and Blade Runner 2049}

\author{
Belén Ramírez Barredo \\ Universidad CEU San Pablo, Madrid \\ belen.ramirezbarredo@ceu.es \\ https://orcid.org/0000-0001-9403-8613
}

\begin{abstract}
Resumen:
La representación de la ciudad ha sido una constante desde los inicios del cine; entre otros motivos, por coincidir su nacimiento con el crecimiento, el desarrollo y la expansión urbanística. El pensamiento político occidental ha perseguido mejorar las condiciones de vida de los habitantes a través del diseño de las metrópolis, una de las principales inquietudes de las utopías. Sin embargo, la ficción cinematográfica nos ofrece habitualmente la creación de imaginarios distópicos que incorporan en su haber un conjunto arquitectónico que responde al pensamiento e ideales de la Arquitectura Moderna.

Este artículo analiza la influencia de estos presupuestos en la representación de la ciudad distópica en el ámbito cinematográfico, a partir de dos enfoques diferentes. De un lado, la proyección de espacios reales como escenario de la distopía (Gattaca, 1997), y, de otro, la construcción de la ciudad distópica a partir de la noción del director sobre la misma (Blade Runner 2049, 2017). Con este fin, se realiza un estudio previo del pensamiento que subyace en los estilos arquitectónicos que emanan de los principios de la Arquitectura Moderna y las posibles representaciones de la urbe en el ámbito cinematográfico, enmarcadas en el contexto histórico sociocultural en que nacieron.
\end{abstract}

\begin{abstract}
:
The representation of the city has been a constant since the beginnings of cinema, among other reasons because its birth coincided with the growth, development and urban expansion. Western political thought has sought to improve the living conditions of inhabitants through the design of metropolises, one of the main concerns of utopias. However, cinematographic fiction usually offers us the creation of dystopian imaginaries that incorporate an architectural ensemble that responds to the thought and ideals of Modern Architecture.

This article analyzes the influence of these assumptions on the representation of the dystopian city in film, from two different approaches. On the one hand, the projection of real spaces as the setting of dystopia (Gattaca, 1997) and, on the other, the construction of the dystopian city from the director's notion of it (Blade Runner 2049, 2017). To this end, a preliminary study is made of the thinking behind the architectural styles that emanate from the principles of Modern Architecture and the possible representations of the city in the cinematographic sphere framed in the socio-cultural historical context in which they were born.
\end{abstract}

Palabras clave: ciudad; cine; distopía; arquitectura moderna; Bauhaus; ciencia ficción.

Keywords: City; Cinema; Dystopia; Modern Architecture; Bauhaus; Science Fiction. 


\section{Introducción}

El proceso de un proyecto arquitectónico se basa en un juego de sentimiento y razón (Zumthor, 2005), adquiriendo "unos valores y significados que están por encima de lo objetivable y lo tangible" (Alba, 2016, p. 125). Alcanza así una doble dimensión: racional y objetiva de un lado; subjetiva y personal de otro. El cine recrea el espacio urbano y, simultáneamente, es un producto industrial, económico y comercial, que influencia e informa la creación de la ciudad por su capacidad de representar, crear imágenes y deseos (Culagovski, 2005). Si bien es cierto que el cine nos acerca las ciudades, la imagen e identidad de las mismas varía en función de la mirada e interés de los cineastas. A través de los recursos básicos de encuadre y montaje, el cine manipula el espacio, influyendo en la construcción de una representación mental "sintética, reducida a hitos aparentemente próximos, fácilmente reconocibles (...) y, naturalmente, sujetos a una posterior explotación comercial” (Gámir, 2012). Así, en muchos casos, "el cine no muestra cómo son las ciudades, sino que se preocupa esencialmente por filmar edificios, construcciones con las que pergeñar un espacio urbano efímero, un escenario evocador de un momento apropiado para el desenlace de una situación dramática concreta” (Ponce, 2011, p. 128). Sin embargo, "la esencia de la Arquitectura debe descubrirse desde las obras concretas” (Kupareo, 1969, p. 2). La elección cinematográfica de un edificio, un espacio urbano o cualquier elemento arquitectónico no es cuestión banal por formar parte de un todo concebido previamente en la mente del director.

El objetivo de la investigación se centra en identificar y analizar las corrientes arquitectónicas, atributos y edificios que los cineastas consideran propios de un imaginario distópico, y evaluar en qué medida la imagen propuesta recoge o distorsiona el pensamiento original subyacente en las obras arquitectónicas, confrontando los principios teóricos de las corrientes con su representación fílmica. Con este fin se ha realizado un análisis metodológico cualitativo en tres fases. En primer lugar, se han localizado los edificios y espacios de rodaje para identificar los estilos arquitectónicos presentes y la filosofía subyacente en su construcción. En segundo lugar, a partir de las locaciones y corrientes identificadas se estudia el imaginario urbano propuesto por cada director. Se finaliza contrastando los 
resultados obtenidos para analizar en qué medida la ciudad física y el pensamiento latente en las corrientes arquitectónicas responde o no a la imagen cinematográfica de las mismas. El estudio requiere una introducción previa sobre la Arquitectura Moderna, movimiento sobresaliente en el siglo XX, y la representación fílmica de la ciudad.

\section{Pensamiento y creación en la Arquitectura Moderna}

La fisonomía de la ciudad es fruto de la historia, el territorio geográfico, las variables económicas o los factores raciales. "La cronología de la evolución arquitectónica en los últimos cien años está marcada por los acontecimientos que cambiaron el rumbo de la historia de toda nuestra civilización" (Sainz, 1997, p. 265): fueron tiempos convulsos, de inestabilidad y descontento social, que generaron un período de gran fecundidad creativa, y que el cine supo recoger a través de propuestas más ambiciosas que el simple entretenimiento de masas (Molina-Siles, 2017).

El Futurismo subrayó la necesidad de fabricar la ciudad como una inmensa obra tumultuosa, ágil, móvil, dinámica en cada una de sus partes, donde la casa futurista sería similar a una gigantesca máquina (Sant'Elia, 1914, Manifiesto de la Arquitectura Futurista). Proyectada sobre escenarios utópicos, la estética Futurista nos trasladó una visión arquitectónica más preocupada por planificar el futuro que por construir el presente (Simon, 2021). El tiempo inicial de las vanguardias manifestó con precisión la crisis existencial del sujeto y su difícil adaptación al sentir de la naturaleza industrial (Fernández, 2001), realidad que plasmó el pensamiento y obra de los principales exponentes de la Arquitectura Moderna. Sus presupuestos quedaron consignados en un concepto de estilo contemporáneo, presente en todo el mundo, unitario e inclusivo, no fragmentado ni contradictorio, al que auguraban larga vida en el siglo XX. Los pioneros de esta corriente impulsaron un cambio radical frente al descrédito provocado por el academicismo, el eclecticismo y a la divergencia de estilos arquitectónicos de finales del XIX (Hitchcock \& Johnson, 1984). El denominado Estilo Internacional se fundamentaba en la "concepción de la arquitectura como volumen más que como masa; en la eliminación de la simetría en favor de la regularidad; y en la proscripción de la decoración aplicada arbitrariamente" (Isac, 2009, p. 37), resultando un 
modo de expresión sin memoria, asimétrico y abstracto, fruto de la acelerada industrialización de las ciudades europeas y norteamericanas, los avances técnicos en la ciencia de los materiales, la mecánica, la ingeniería y la construcción (Martínez, 2018). La estética en el ámbito arquitectónico abarcó todos los aspectos de la vida cotidiana, recogidos en los principios formulados por Mies van der Rohe -"Menos es más"- y Louis Sullivan -"La forma sigue a la función"-.

La Bauhaus, más que un estilo arquitectónico, representó una nueva forma de pensar (Cook, 2017). En su primera etapa, se desarrollaron una serie de corrientes acordes con los principios de la Arquitectura Moderna: líneas puras, ausencia de ornamentación, empleo de nuevos materiales y búsqueda de estructuras que liberasen la masa de los muros, de forma que la belleza no fuese producto de la decoración, sino fruto del conjunto diseñado (Martínez, 2018). El Neoplasticismo se caracterizó por el empleo de líneas horizontales y verticales, ángulos rectos y superficies de colores, su sentido universalista y el deseo de unir el arte con la vida (Serra, 2010). El Cubismo se centró en la geometría y la exploración tridimensional del espacio, con una estética de cubos simples y superpuestos, sin desniveles. El Organicismo nació de la concepción de la Arquitectura como un todo, promoviendo la armonía entre el hábitat humano y el mundo natural (Martínez, 2018). Si bien arrancaba de las premisas del racionalismo, se distanció del mismo en algunos aspectos: los espacios no constituían una máquina para vivir y, lejos de todo signo de estandarización, la estructura y apariencia de un edificio debía basarse en formas orgánicas y armonizar con su entorno natural. Frank Lloyd Wright, máximo exponente americano de este pensamiento, entendió la arquitectura desde un enfoque personalista, como servicio al hombre, afirmando que "la forma y la función son una" (Greelane, 2019). En contraposición, el Funcionalismo supeditó la forma de los edificios a su función. Entre los defensores de esta pragmática línea arquitectónica están Sullivan y Le Corbusier. La concreción de sus proyectos urbanísticos se tradujo en ciudades terriblemente monótonas; la construcción de edificios económicos degeneró en viviendas de baja calidad que propiciaron barrios residenciales donde acamparon la disgregación social, la violencia y la delincuencia (Martínez, 2018). Entre 1950 y 1970, tuvo su auge el Brutalismo o Arquitectura Brutalista: inspirado en el trabajo de Le Corbusier y Eero Saarinen, se caracterizó por el empleo masivo del concreto como material de construcción, con el fin de resaltar el poder de la estructura en su conjunto 
(Alejandro, 2021). Su arquitectura simple y repetitiva encontró gran aceptación en la antigua Unión Soviética. Alrededor de los años sesenta, surgió el Formalismo, dominado por la austeridad y la consideración de la forma como principio en sí mismo, independiente de su función. En torno a los años 60-80, "como respuesta a las contradicciones de la Arquitectura Moderna, y en especial a los postulados del Estilo Internacional" (Martínez, 2018), despuntó la Arquitectura Postmoderna con el fin de contrarrestar la austeridad, la formalidad y la falta de variedad, al considerar que la ornamentación y las alusiones históricas añaden riqueza a la arquitectura (Venturi, 1966). A finales de la década de 1990, este concepto se transformó en un término genérico que engloba multitud de nuevas tendencias, entre las que se encuentran la Arquitectura de Alta Tecnología, Neofuturista y Deconstructivista.

\section{Representación de la ciudad moderna en el cine}

La representación de la ciudad en el cine ha sido, históricamente, una vía para señalar cuestiones de ámbito social, político y cultural. Las primeras películas de los hermanos Lumière, testigos objetivos de la vida cotidiana, exhiben escenas de la vida urbana a modo de documental. Estos inicios del séptimo arte llevaron a Michel Marie a definir el cine como "una invención urbana", porque "nació en las afueras de Lyon e hizo su debut en los grandes bulevares de París" (Antoniazzi, 2018). Afirmación refrendada por los hechos: "de las 1424 cintas que forman el catálogo Lumière, más de tres cuartos tienen como tema o escenario principal la ciudad" (Mottet, 2011, p. 135). Como afirma Weihsmann: "Ever since the beginning of cinematography in the early 1900 s, filmmakers of all film genres (...) have been fascinated with the topography and image of the modern metropolis" [Desde los comienzos de la cinematografía, a principios del siglo XX, los cineastas de todos los géneros (...) se han sentido fascinados por la topografía y la imagen de las metrópolis modernas] (Weihsmann, 2011, p. 25).

El origen del cine coincidió con el crecimiento demográfico, la expansión geográfica y el desarrollo económico urbano. Durante el siglo XIX, las capitales europeas experimentaron un crecimiento sin precedentes, especialmente notable en el caso de Londres y Berlín, propiciado, en gran medida, por dos factores: la expansión colonial y la irrupción de la corriente eléctrica. El primero amplió la 
geografía económica de Europa, reclamando una nueva distribución del espacio; el segundo favoreció el crecimiento periférico de población en torno al núcleo urbano, en asentamientos que concentraban una densa mano de obra inmigrante junto a grandes instalaciones industriales, en condiciones insalubres. Las tensiones y conflictos de tipo social y político derivados del desarrollo industrial condujeron a un interés creciente, por parte de los poderes públicos, en el ámbito urbanístico, provocando profundos cambios en la morfología urbana. En este contexto, emerge el concepto de metrópoli, entendido en términos de área urbana de grandes dimensiones, "un todo orgánico al que los individuos se tienen que enfrentar y en el cual, dependiendo de las circunstancias, llevan una vida grata o difícil” (Sorlin, 2001).

En las metrópolis, símbolos de la modernidad, "surgen nuevos espacios de sociabilidad y de consumo, que el cine retratará en numerosas películas" (Antoniazzi, 2018, p. 10): un espacio tejido por conflictos, nuevas relaciones e intercambio de vivencias, donde la realidad "was no longer perceptually experienced as a smooth, spatiotemporal continuum, but rather as dynamic and fragmented, as constantly changing and susceptible to violent rupture" [ya no se percibe como un continuo espacio-temporal suave, sino como algo dinámico y fragmentado, en constante cambio y susceptible de una violenta ruptura] (Turvey, 2011, p. 56). Como afirma Lynch, "moving elements in a city, and in particular the people and their activities, are as important as the stationary physical parts" [los elementos móviles de una ciudad, y en particular las personas y sus actividades, son tan importantes como las partes físicas fijas] (Lynch, 1960, p. 2). El cinematógrafo de los hermanos Lumière centró sus esfuerzos en recoger la realidad objetiva, el fluir incesante de las multitudes y trasladar ese movimiento al espectador. Filmaron ajustándose a un punto de vista frontal e inmóvil y a una única toma, pero "as cinemathic techniques developed, films increasingly drew on camera mobility and editing devices to deliver their messages, these movements were no longer necessarily objective" [a medida que se desarrollaban las técnicas cinematográficas, las películas recurrían cada vez más a la movilidad de la cámara y a los dispositivos de montaje para transmitir sus mensajes, estos movimientos ya no eran necesariamente objetivos] (Kracauer, 196o, p.34). 
Frente a la evolución natural que experimentan las ciudades, el cine posee el poder de detener el tiempo. No obstante, a través de las películas solo podemos alcanzar una visión parcial y fragmentaria que, además, varía según los lugares y atributos seleccionados por los cineastas (Antoniazzi, 2019).

El papel que desempeña la ciudad difiere en función de la historia, la visión que nos proporciona el director, la trama que en ella se desarrolle, el modo en que la vivan los protagonistas o el género cinematográfico. Hay historias que se desarrollan en la ciudad, y otras en que la ciudad es protagonista de la narración. Relatos donde el entramado urbano supera la condición de mero territorio físico, convirtiéndose en generador de una atmósfera que envuelve, condiciona los conflictos, los personajes y las historias que en ella suceden. (Ramírez, Izquierdo \& Alcaraz, 2019)

Cuando el papel de la urbe se reduce a representar el espacio físico geográfico donde se desarrolla la trama, hablamos de la ciudad como escenario. Se ofrece una perspectiva limitada a un encuadre teórico y abstracto, un decorado para la acción, con entidad vaga, marcada por los tópicos. Hasta la década de los sesenta los retratos cinematográficos de la ciudad se limitaban a mostrar una serie de espacios y edificios emblemáticos, acercándose más a un modelo que un lugar real (Sorlin, 2001). Un ejemplo es Nueva York, referente entre las grandes metrópolis (Lus, 2016). La ciudad-ficción por excelencia debe su forma actual a los avatares del mercado e iniciativas privadas, que triplicaron entre el año 1950 y final de siglo el número de rascacielos que se incorporaron al ya recurrente imaginario simbólico de la ciudad. Una idea un tanto lejana de la realidad de Nueva York, donde los distritos comerciales y de oficinas, cuajados de rascacielos, significan tan solo el $4 \%$ de la ciudad, frente a un conjunto arquitectónico que no rebasa los 20 metros de altura (Durán, 2017).

Cuando la ciudad supera la condición de espacio físico y adquiere un rol propio empezamos a cuestionarnos el concepto como tal. En el imaginario cinematográfico es necesario tener presente que la arquitectura que reflejan las ciudades no se considera sólo como un espacio habitable, sino como un espacio cinematográfico por sí solo (Reyes, 2013). En el ámbito de la ciencia-ficción, las películas "pueden terminar teniendo más sustancia que la mayoría de los otros géneros populares" (Telotte, 2002, p. 19), ya que la representación de la urbe busca "inspira- 
ción en una miríada de influencias arquitectónicas" (Hanson, 2006, p.8). La sociedad y el individuo son los agentes que actúan de forma inherente en la construcción progresiva de un espacio, que formulan según sus criterios y respectivas representaciones del mundo, transformándolo en una manifestación de la sociedad constructora (Vergara, 2014), un emplazamiento real tejido de encuentros y vivencias humanas que dan vida al concepto de metrópoli. Como indica Simmel, "la ciudad no es una entidad espacial con consecuencias sociológicas, sino una entidad sociológica que se forma espacialmente" (Frisby \& Featherstone, 2000, p.11). En este sentido, Metrópolis (Metropolis, Fritz Lang, 1927), inspirada en la ciudad de Nueva York, "no se limita a la simple visión monumental o la estilización estética de la ciudad industrial como reino sublime"; el relato que discurre entre los muros de la gran ciudad "reúne, en realidad, dos ciudades confrontadas" que reflejan "un conflicto humano en el que se entretejen los aspectos psicológicos, sociales, tecnológicos y morales que configuran la compleja trama de la narración fílmica” (Subirats, 1986).

\section{Arquetipo de la ciudad del futuro: análisis de Gattaca y Blade Run-} ner 2049

Una vez introducida la evolución y principios de la Arquitectura Moderna y la representación fílmica de la metrópoli, abordamos el arquetipo de ciudad en Gattaca (Andrew Niccol, 1997) y Blade Runner 2049 (Denis Villeneuve, 2017). En primer término, debemos recordar que toda narración se encuadra en un marco espacio-temporal consustancial al relato. El escenario delimita cierta cinematografía de género y puede ser natural, o construido artificialmente en estudios (Vilches, 2017). En cualquier caso "una imagen ambiental puede analizarse en tres componentes: identidad, estructura y significado" (Lynch, 1990, p. 8), de modo que el análisis no se centra tanto en la ciudad física como en la imagen mental que los ciudadanos extraen de ella.

Gattaca y Blade Runner 2049 proyectan una visión distópica de la sociedad donde la crítica humanista "intenta comprender la naturaleza humana y el lugar de la humanidad en el esquema de las cosas, planteando las cuestiones tradicionales: ¿quiénes somos? Y ¿cuál es el sentido de la vida?” (Bywatter \& Sobchack, 
como se citó en Telotte, 2002, p. 46). Con dos décadas de diferencia, ambos largometrajes fusionan el género de ciencia-ficción y el cine negro, en una propuesta estética y visual de la ciudad de Los Ángeles muy diferente. Mientras Gattaca se filmó en esta locación y sus alrededores, la proyección de Blade Runner 2049 fue levantada ex profeso como decorado real a partir de las indicaciones del director.

\subsection{Análisis arquitectónico de los emplazamientos y edificios de Gat- taca y Blade Runner 2049.}

Gattaca (1997), haciendo gala de una estética retro-futurista, la distopía se articula en torno a la eugenesia, esto es, la mejora de los rasgos hereditarios mediante la intervención manipulada y métodos selectivos. La suplantación de la identidad de un hombre genéticamente superior por parte de un no válido, con el fin de cumplir su sueño de viajar al espacio (IMDb, s.f.), se despliega en un imaginario neutro, carente de personalidad, espejo de una sociedad competitiva, cruel y deshumanizada.

Gattaca se rodó en la ciudad de Los Ángeles (California), y emplazamientos cercanos donde se ubican edificaciones representativas de la Arquitectura Moderna (IMDb, 1997). La corporación Gattaca es el Marin County Civic Center, sito en San Rafael, obra póstuma de Lloyd Wright. Se levantó a partir de una serie de bocetos donde el arquitecto reflejaba sus aspiraciones y fantasías sobre la ciudad futurista (Altamirano, 2014). Su objetivo era crear un espacio accesible, cómodo, familiar y luminoso. Resumía su concepción sobre la misión del arquitecto en "ayudar a la gente a entender cómo hacer que la vida sea más bella, que el mundo sea mejor para vivir, y dar razón, rima y sentido a la vida" (Lloyd Wright, 1957, como se citó en Frank Lloyd Wright Foundation, s.f.). Acorde con la corriente Orgánica que propugnó, el edificio participa del entorno natural, adaptándose a las condiciones circundantes.

Los bloques de viviendas residenciales pertenecen a la sede de la Universidad Politécnica del Estado de California, en Pomona. Destaca el CLA Building, de estilo futurista, obra del arquitecto Antoine Predock. Para él "Architecture is about deeply and authentically connecting to the essence. Each building should be a singular, unique event locked in time and place, and at the same time a launchpad into the future" [la arquitectura consiste en conectar profunda y auténticamente 
con la esencia. Cada edificio debe ser un acontecimiento singular, único, encerrado en el tiempo y el lugar, y al mismo tiempo una plataforma de lanzamiento hacia el futuro]. Su concepción de la arquitectura es la de un encuentro poético: "Any building must have a life of its own, independent of the program, but of course, accommodating the client's needs. When architecture becomes simply program-driven, merely a functional diagram, it is soulless" [Cualquier edificio debe tener vida propia, independiente del programa, pero por supuesto, acomodándose a las necesidades del cliente. Cuando la arquitectura se convierte en un simple programa, en un mero diagrama funcional, carece de alma] (Pedrock en Belogolovsky, 2020).

El departamento de policía se emplaza en el Otis College of Art and Design. Concebido por Eliot Noyes, fue diseñado para albergar la sede aeroespacial de IBM. Su trabajo se inspiró en los principios de Gropius, Breuer y Le Corbusier, erigiéndose en firme defensor del estilo funcional y moderno. Abogó por la simplicidad de la forma y la naturaleza de los materiales.

The Forum, obra del arquitecto Charles Luckman, representa el papel de laboratorio de detección de ADN. "Luckman aportó a la arquitectura su perspicacia empresarial y su talento para el espectáculo". Convencido de "que la arquitectura es un negocio y no un arte", destacó por el enfoque pragmático de sus obras, y, pese a afirmar" que sus edificios no se ajustan a un solo estilo arquitectónico, se adhiere estrechamente al credo funcionalista del diseño moderno" (Muschamp, 1999).

Los Angeles City Hall fue diseñado por J. Parkinson, A. C. Martin y J.C. Austin. Inaugurado en 1928, incorpora diferentes elementos, algunos de corte clásico y Art Decó, que devienen en una obra híbrida única. Esta locación, perteneciente según Austin al estilo "Americano Moderno", alberga la filmación del concierto de piano.

Los Angeles Theatre, proyectado por A. C. Martin Sr. y W. Lee Woollett, alberga en la película el Restaurante Lavish. El edificio, con una fachada de estilo churrigueresco, goza del primer balcón de hormigón armado en voladizo del mundo.

Otras localizaciones: la Presa de Sepúlveda (Encino, Los Ángeles), edificada por el Cuerpo de Ingenieros del Ejército de Estados Unidos; la Kramer Junction (Ca- 
lifornia) representa el papel de planta solar; el Culver City High School (California), como pre-school; el túnel de 2nd Street, entre Hill y Figueroa (Los Ángeles) y la playa de La Jolla (San Diego).

Blade Runner 2049 (2017), acorde con el estilo de las obras de Villeneuve, transita por espacios comunes donde los protagonistas $-\mathrm{K}$, un joven Blade Runner y Deckard, un antiguo agente de ese mismo cuerpo que lleva 30 años desaparecido (IMDb, s.f.) - se sitúan en una encrucijada vital, atrapados entre la búsqueda constante de su propia identidad y una realidad asfixiante, escenificada en el cambio climático y su consecuente impacto sobre la arquitectura urbana. Pese a dejar que sea "el espectador quien extraiga sus propias conclusiones del relato" (Gómez, 2021, p. 134), el director traslada al mismo su visión sobre la sociedad contemporánea y sus experiencias personales (Gómez, 2021).

Blade Runner 2049 se filmó en Budapest, Hungría (IMDb, 2017). A diferencia de Gattaca, su producción se desarrolló fundamentalmente en el plató exterior de Origo Film Studios (Budapest) y en tres decorados de Korda Studios en Etyek (Hungría).

Entre los edificios existentes, destaca el Stock Exchange Palace. Construido en 1905 para albergar la Bolsa de Valores y Mercancías de Budapest, el gran edificio Beaux Arts fue diseñado por Ignacz Alpar en el distintivo estilo Art Nouveau de la Secesión húngara. En el filme se utiliza para recrear el casino en ruinas de Las Vegas.

El exterior del edificio de la vivienda de K se sitúa en el cruce de las calles Szalay y Honvéd (Budapest). Para recrear el orfanato del Señor Cotton, se utilizó el interior de la antigua planta nuclear soviética de Inota, en Várpalota, y un antiguo almacén de electrónica de Kistarcsa, en las afueras de Budapest.

Los primeros minutos de la cinta exhiben planos aéreos de la planta termosolar Gemasolar de Fuentes de Andalucía (Sevilla). Así mismo, en el transcurso del filme podemos contemplar vistas aéreas de los Invernaderos del Poniente Almeriense y de Islandia. Otras locaciones corresponden a tomas aéreas de las favelas de Ciudad de México, utilizadas para recrear la ciudad de Los Ángeles, y otras del Parque Estatal Valle del Fuego (Nevada) para idear los alrededores de Las Vegas. 
El interior de la oficina de Wallace se inspira en el proyecto presentado por el estudio español de arquitectos Barozzi Veiga en 2010 para el diseño del Museo del Neardental de Piloña, España.

\subsection{Análisis de la ciudad en Gattaca y Blade Runner 2049}

Gattaca se filmó en diferentes locaciones de la ciudad de Los Ángeles, en escenarios ambientados en construcciones racionalistas de líneas nítidas y predominantemente rectas, que acentúan el carácter frío y aséptico del mundo de Gattaca (Belucci, 2014). A través de planos generales dominados por la simetría o la filmación de escenas con un horizonte integrado por edificios de gran escala de concreto aparente con estructuras de acero cromado, el director transmite la frialdad y escasa relación que existe entre sus miembros. El minimalismo, la geometría, la ausencia de texturas y la inexistencia de referencias a cualquier canon clásico o histórico, construyen una ciudad atemporal, abstracta, sin memoria, carente de emoción.

Racionalidad y funcionalidad son la clave para entender la arquitectura en una sociedad que mide y valora a las personas por su rendimiento y supuesta perfección. La cinta incorpora edificios emblemáticos pertenecientes a diferentes corrientes de la Arquitectura Moderna (Orgánica, Funcionalismo, Formalismo) y Postmoderna, si bien, el encuadre y montaje distorsionan el espacio físico y formal. Frente a la concepción personalista y orgánica de Lloyd sobre el Marin County Civic Center, la sede de Gattaca comunica el pragmatismo propio del Funcionalismo, simbolizando el principio de máximo rendimiento y perfección dominante en el filme, donde la arquitectura se concibe como una máquina para trabajar. Un ejemplo en este sentido es la disposición del mobiliario de trabajo, que transforma el espacio en un lugar donde el hombre adopta un comportamiento autómata, evocando las incipientes oficinas de los años cuarenta. El director pone el acento en la geometría, símbolo del pensamiento racional y la perfección, y en la repetición de elementos, signo del anonimato, la uniformidad y el mecanicismo. Ejemplo de la primacía de la función sobre la forma es la disposición del auditorio en el concierto de piano, en torno a un escenario circular. Cabe destacar el simbolismo que encierra el diseño de la escalera con forma de doble hélice en el apartamento de Eugene. 
Blade Runner 2049 fue filmada en Budapest, ciudad escogida por la diversidad estética que encierra. En ella se entremezcla la arquitectura propia de Europa del Este con estructuras brutalistas de la época soviética. Para trabajar en entornos auténticos, se construyeron decorados reales en Origo Film Studios y Korda Studios siguiendo las directrices de Villeneuve. La estética arquitectónica brutalista unifica el contenido a lo largo del filme. Un estilo concebido para representar el mundo moderno, igualitario y utópico. Con origen en la arquitectura Moderna, bebe de la filosofía positivista de Comte, que conduce a la negación del individuo en aras de una Humanidad abstracta. En Blade Runner 2049 no existen estructuras históricas, emplazándonos en un futuro homogéneo, sin personalidad.

El interior del departamento de $\mathrm{K}$ alberga cierta semejanza con el proyecto de apartamentos para trabajadores diseñado por Walter Gropius; un ejemplo de la vivienda mínima propulsada por algunos arquitectos modernos, imagen de la construcción de edificios residenciales económicos. Entre el hacinamiento de monumentales rascacielos y edificios de concreto, homogéneos y repetitivos, destaca el muro de hormigón que protege a la ciudad frente al acoso del océano. El interior futurista de las oficinas y archivos de la Wallace Corporation contrastan con el diseño aséptico y minimalista de la oficina de Wallace, interpretación del proyecto original del estudio Barozzi Veiga. Tan solo el interior del Palacio de la Bolsa, convertido en casino de Las Vegas, actúa como nexo con el pasado: un edificio con personalidad que deja de lado el anonimato para formar parte del presente del espectador.

\subsection{Análisis comparativo del arquetipo ciudad en Gattaca y Blade Runner 2049}

Gattaca fue rodada en la ciudad de Los Ángeles. Pese a incorporar edificios icónicos de la Arquitectura Moderna, su estética retrofuturista concede a la ciudad una belleza impersonal y, en cierta medida, atemporal. El espacio geográfico y la relación entre los diferentes enclaves de la ciudad, entendido como escenario donde se desarrolla la trama, no es relevante en el filme. Todo recorrido, edificio, espacio o lugar capturado por la cámara posee un significado más profundo, dirigido a reforzar el sentimiento de deshumanización, frialdad y pérdida de individualidad latente en el filme. El encuadre y el montaje manipulan el espacio de 
la ciudad, de modo que los edificios considerados de forma individual y su filosofía originaria pierden relevancia frente a la exposición de la ciudad como un todo. Blade Runner 2049 emplaza al espectador a la ciudad de Los Ángeles en un futuro no lejano, si bien la filmación de espacios tuvo lugar en Budapest y se complementó con la construcción de decorados reales a partir de la imagen de metrópoli que el director perseguía mostrar. Villeneuve pone el acento en el estilo brutalista, integrado por edificios homogéneos, sin color, ni personalidad; una ciudad muerta pero intensa, superpoblada y sucia, sin identidad, abarrotada de hologramas, publicidad personalizada y luces de neón, donde la vivienda futurista, dominada por la tecnología, acentúa la soledad y el imperio de la colectividad sobre el individuo.

\section{Conclusiones}

En la base del imaginario arquitectónico distópico, proyectado en ambos largometrajes, encontramos los principios latentes en la arquitectura Moderna. La primacía de la racionalidad y funcionalidad arquitectónica, la ausencia de ornamentación, la uniformidad, el predominio del funcionalismo y el exceso de minimalismo ahogan los signos de expresión e identidad del individuo. Este es relegado a un segundo plano bajo la supremacía de la colectividad. Los atributos característicos de la Arquitectura Moderna se identifican cinematográficamente con el hábitat propio de sociedades distópicas. El pragmatismo de la Arquitectura Funcional, lejos de promover el desarrollo personal, suscita ciudades monótonas, donde prima la construcción de edificios económicos de baja calidad. El estilo brutalista, de corte simple y repetitivo, erige ciudades asépticas, sin alma ni personalidad.

En las distopías aquí analizadas, la ciudad ocupa un lugar indirecto, por no ser el enemigo al que se enfrenta el individuo. No obstante, el concepto del espacio como manifestación de la sociedad constructora, lejos de ser una realidad positiva, emplaza al ciudadano en el anonimato y la indiferencia.

El encuadre y montaje manipulan el espacio de la ciudad y desdibujan la mirada original del arquitecto sobre su obra. La disposición compositiva de los planos acentúa la magnitud de los edificios frente al individuo, trasladando al espectador 
la escasa relevancia de los sentimientos personales ante el poder de la urbe entendida como colectividad.

Aunque la representación visual de la ciudad en Gattaca y Blade Runner 2049 varía, entre otros factores, en función de la trama o visión del director, los dos filmes coinciden en la imagen que transmiten de la misma, independientemente de su filmación en un espacio real o construido. Ambos describen una sociedad que habita en situaciones indeseables, consecuencia de un espacio construido por la propia humanidad a través de decisiones y comportamientos sociales de gran actualidad: la manipulación genética, el cambio climático, el consumismo, la sobreexplotación de los recursos, etc.

\section{Discusión}

El origen del cine enlaza con el crecimiento, desarrollo y expansión de la ciudad moderna. Su representación en el ámbito fílmico varía en función de numerosos factores, si bien la imagen que se ofrece es siempre parcial y condicionada por la mirada del director. En palabras de Vielma: "El cine como expresión estética propia del siglo XX, y como la elaboración más desarrollada y difundida de una experiencia del tiempo y el espacio, continúa ofreciendo representaciones de mundos posibles" (2019, p. 72).

La evolución arquitectónica de la ciudad y su consiguiente fisonomía "está marcada por los acontecimientos que cambiaron el rumbo de la historia de toda nuestra civilización" (Sainz, 1997, p. 265) en la Edad Contemporánea. Las Vanguardias, ámbito al que se acercaron los principales exponentes de la Arquitectura Moderna, plasmaron la crisis existencial y difícil adaptación de la sociedad al sentir de la naturaleza industrial.

La Bauhaus fue mucho más que un estilo arquitectónico: representó una nueva forma de pensar (Cook, 2017). Las corrientes que nacieron al calor de la misma se inspiraron en los principios de racionalidad y funcionalidad, propiciando construcciones estandarizadas, repetitivas, asépticas, sin identidad, imagen y espejo cinematográfico de la metrópoli distópica. 
Los presupuestos de la Arquitectura Moderna están influenciados por la filosofía positivista, en la que el individuo es relegado a un segundo plano frente al concepto Humanidad como colectivo. El estilo brutalista, que se propagó en los países de la antigua Unión Soviética, es un ejemplo. Algunas corrientes nacidas en el primer período de la Bauhaus, como el Organicismo, entendieron la arquitectura en términos de servicio al hombre. Sin embargo, los principios “menos es más” y "la forma sigue a la función" primaron, en gran medida, en los diferentes estilos arquitectónicos del siglo XX.

En el ámbito fílmico, la ciudad puede exhibirse como decorado construido a partir de tópicos donde discurre la trama; o bien puede revelar la ciudad vivida, es decir, aquella que se construye a través de las diferentes relaciones que se entablan en el tejido social, siendo la ciudad el espacio que surge de las relaciones humanas entre sí y con su entorno. La ciudad distópica retrata la ciudad construida, resultado de las relaciones de la sociedad con su espacio y entre sí. La deshumanización característica del imaginario distópico se traduce en la imagen de metrópoli como fruto de la sociedad constructora.

\section{Referencias bibliográficas}

Alejandro, L. (2021). ¿Qué es la Arquitectura Brutalista? El culto máximo al hormigón. Moovemgazine.com. Recuperado de https://moovemag.com/2019/08/que-es-la-arquitectura-brutalista-culto-al-hormigon/

Altamirano, R. (2014). Cine y Arquitectura: Gattaca. Plataforma Arquitectura. Recuperado de https://www.plataformaarquitectura.cl/cl/o2336854/cine-y-arquitectura-gattaca-2

Antoniazzi, S. (2019). La ciudad filmada: cine, espacio e historia urbana. Biblio3W. Revista Bibliográfica de Geografia y Ciencias Sociales, Vol. XXIV (1.260), 1-24. Recuperado de https://revistes.ub.edu/index.php/b3w/article/view/27278

Alba Dorado, M. I. (2016). Arquitectura y creatividad. Reflexiones acerca del proceso creativo del proyecto arquitectónico. Arquitectura Revista, 12(2), 125-139.

Belogolovsky, V. (2020). "My Buildings Are Rides": In Conversation with Antoine Predock. Archdaily. Recuperado de https://www.archdaily.com/941725/my-buildings-are-rides-in-conversation-with-antoinepredock

Belucci, G. (2014). "Un futuro no muy lejano”... Gattaca revisitada. Aesthethika. Revista internacional de estudio e investigación interdisciplinaria sobre 
subjetividad, política $y$ arte, 10(1), 62-76. Recuperado de https://www.aesthethika.org/Un-futuro-no-muy-lejano-Gattaca

Cook, W. (2017). El secreto del éxito de la Bauhaus, la escuela perseguida por los nazis en Alemania que revolucionó la arquitectura y el diseño. BBC News. Recuperado de https://www.bbc.com/mundo/vert-cul-42096241.

Culagovski, R (2005). El cine como recreador de ciudades. laFuga, 1.

Durán Fernández, J. (2017). Nueva York 1916. La ciudad sin límites | New York 1916. Unlimited city. ZARCH, (8), 90-103. https://doi.org/10.26754/ojs zarch/zarch.201782148.

Fernández Alba, A. (2001). El pensamiento arquitectónico del siglo XX. Aula Abierta sobre El pensamiento arquitectónico del siglo XX. Ciclo conferencias Fundación Juan March. Recuperado de http://recursos.march.es/culturales/documentos/conferencias/resumenesbif/128.pdf

Frank Lloyd Wright Foundation (s.f.). About Frank Lloyd Wright. franklloydwright.org. Recuperado de https://franklloydwright.org/franklloyd-wright/

Frisby, D. \& Featherstone, M. (2000). Simmel on Culture: Selected Writings. London: Sage Publications.

Gámir, A. (2012). La consideración del espacio geográfico y el paisaje en el cine. Scripta Nova. Revista Electrónica de Geografía y Ciencias Sociales, XVI(403). Recuperado de www.ub.es/geocrit/sn/sn-403.htm

Gómez, P. (2021). Villeneuve. La mirada salvaje. Palma de Mallorca: Dolmen Editorial.

Greelane (2019). Arquitectura orgánica: Frank Lloyd Wright a la interpretación modernista. Greelane.com. Recuperado de https://www.greelane.com/es/humanidades/artes-visuales/organic-architecture-nature-as-a-tool-178199/

Hanson, M. (2006). Cine digital. Escenarios de Ciencia Ficción. Barcelona: Editorial Océano.

Hitchcock, H. R. \& Johnson, P. (1984). El Estilo Internacional. Arquitectura desde 1922. Colegio Oficial de Aparejadores y Arquitectos Técnicos. Murcia: Galería-Librería Yerba.

IMDb (s.f.). Gattaca, 1997. Filming \& Production. Recuperado de https://www.imdb.com/title/tto119177/locations?ref $=$ tt ql sm

IMDb (s.f.). Blade Runner 2049, 2017. Filming \& Production. Recuperado de https://www.imdb.com/title/tt1856101/locations?ref =tt ql sm\#filming locations

Isac Martínez de Carvajal, A. (2012). La Historia de la Arquitectura del siglo XX: modelos historiográficos. En I. Ruiz de la Peña González (res.), Lecciones de los maestros: aproximación histórico-crítica a los grandes historiadores de la arquitectura española. De arte: revista de historia del arte (11), 3558 . 
Jiménez González, M. \& Latorre Izquierdo , J. (2020). La crisis constructiva del sueño europeo en Metrópolis, de Fritz Lang. Un estudio contextual comparado de los mitos de Prometeo y Atlas. Fotocinema. Revista Científica De Cine y Fotografía, (21), 83-110. https://doi.org/10.24310/Fotocinema.2020.vi21.10000

Kracauer, S. (1960). Theory of film: The Redemption of Physical Reality. Nueva York: Oxford University Press.

Kupareo, R. (1969). Filosofía de la Arquitectura. AISTHESIS: Revista Chilena de Investigaciones Estéticas, (4), 21-32.

Lus Arana, L. M. (2016). Nueva York, al otro lado del espejo. El cine y la ciudad futura como texto / New York, through the looking-glass. Cinema and the future city as a text. Proyecto, Progreso, Arquitectura, (14), 40-53. https://doi: 10.12795/ppa.2016.114.033

Lynch, K. (1990). The image of city. Cambridge: M.I.T. Press.

Marcos Ramos, M. (2018). Cine y ciudad. Condenadas a Entenderse. Fonseca, Journal of Communication, (16), 189-192. Recuperado de www.revistas.usal.es/index.php/2172-9077/article/view/18543

Molina-Siles, P. (2017). Dibujando Metrópolis. El sueño arquitectónico de Fritz Lang. EGA Expresión Gráfica Arquitectónica, 22(29), 190-199. https://doi: 10.4995/ega.2017.5229

Mottet, J. (2011). Elementos para una genealogía del paisaje estadounidense (1897- 1912). En G. P. Brunetta (Ed.), Historia mundial del cine, vol. 1, tomo 1 (pp. 135-160). Madrid: Akal.

Muschamp, H. (1999). Charles Luckman, Architect Who Designed Penn Station's Replacement, Dies at 89. The New York Times. Recuperado de https://www.nytimes.com/1999/01/28/arts/charles-luckman-architectwho-designed-penn-station-s-replacement-dies-at-89.html

Ponce Herrero, G. (2011). Futuro imperfecto: las ciudades del mañana en el cine. Boletín de la Asociación de Geógrafos Españoles, (55), 127-152.

Sant'Elia, A. (1914). La Arquitectura Futurista. Manifiesto. Recuperado de https://www.uclm.es/centros-investigacion/cdce/sintitulo/sin-numero6/sin6-arquitecturafuturista/1-elia

Ramírez, B., Izquierdo, V. \& Alcaraz, A. (2019). La imagen de marca de la ciudad a través de los títulos de crédito. Actas Icono 14, 1(1), 124-147. Recuperado de https://icono14.net/ojs/index.php/actas/article/view/1291

Reyes Vázquez, J. C. (2013). La ciudad automática: imaginario urbano en el cine de ciencia ficción. CIENCIA ergo-sum, Revista Científica Multidisciplinaria de Prospectiva, 20(1), 53-60. Recuperado de https://www.redalyc.org/articulo.oa?id=10425466008

Sainz Avia, J. (1997). Arquitectura y urbanismo del siglo XX. En J. A. Ramírez (Ed.), Historia del arte, volumen 4. El mundo contemporáneo (pp. 265335). Madrid: Alianza Editorial. doi: 10.13140/RG.2.1.1235.2248

Serra Lluc, J. (2010). La versatilidad del color en la composición de la arquitectura contemporánea europea: contexto artístico, estrategias plásticas $e$ intenciones (Tesis doctoral). Universidad Politécnica de Valencia. 
Simon (2021). Arquitectura futurista y estética computacional. Simonelectric.com. Recuperado de https://www.simonelectric.com/detailers/arquitectura-futurista-y-estetica-computacional.

Sorlin, P. (2001). El cine y la ciudad: una relación inquietante. Secuencias: revista de historia del cine (13), 21-28.

Subirats, E. (1986). La ciudad del fin del mundo. Los Cuadernos del Norte: Revista cultural de la Caja de Ahorros de Asturias, 7 (39), 24-33.

Telotte, J. P. (2002). El cine de ciencia ficción. Madrid: Cambridge University Press.

Vilches Manterola, L. (2017). Diccionario de teorías narrativas. Barcelona: Caligrama.

Turvey, M. (2011). The Filming of Modern Life: European Avant-Garde Film of the 1920s. Cambridge: MIT Press.

Venturi, R. (1966). Complexity and Contradiction in architecture. New York: Museum of Modern Art.

Vergara, F. (2014). El cine, una herramienta para la comprensión geográfica. $R e$ vista Geográfica de Valparaíso, (49), 80-97.

Vielma Cabruja, J. I. (2019). Tres ciudades en el cine de ciencia ficción. Distopías y consolidación de un régimen de acumulación flexible. Estoa, 16(8), 7181. https://doi: 10.18537/est.voo8.no16.ao6

Weihsmann, H. (2011). Ciné-City Strolls: Imagery, Form, Language and Meaning of the City Film. En F. Penz \& A. Lu (Eds). Urban Cinematics: Understanding Urban Phenomena Through the Moving Image. Bristol: Intellect.

Zumthor, P. (2009). Pensar la arquitectura. Barcelona: Gustavo Gili. 